\title{
Ca II K observations of QSOs in the line-of-sight to the Magellanic Bridge ${ }^{\star}$
}

\author{
J. V. Smoker ${ }^{1,2}$, F. P. Keenan ${ }^{1}$, H. M. A. Thompson ${ }^{1}$, C. Brüns ${ }^{3}$, E. Muller ${ }^{4}$, N. Lehner ${ }^{5}$, \\ J.-K. Lee ${ }^{1}$, and I. Hunter ${ }^{1}$
}

\author{
1 APS division, Dept. of Pure and Applied Physics, Queen's University, University Road, Belfast, BT7 1NN, UK \\ e-mail: j.smoker@qub.ac.uk \\ 2 European Southern Observatory, Alonso de Cordova 3107, Casilla 19001, Vitacura, Santiago 19, Chile \\ 3 Radioastronomisches Institut, Universität Bonn, Auf dem Hügel 71, 53121 Bonn, Germany \\ 4 Australia Telescope National Facility, CSIRO, PO Box 76, Epping N.S.W, 1710, Australia \\ 5 Department of Astronomy, University of Wisconsin, 475 North Charter Street, Madison, WI 53706, USA
}

Received 15 May 2005 / Accepted 28 July 2005

\section{ABSTRACT}

We describe medium-resolution spectroscopic observations taken with the ESO Multi-Mode Instrument $(\mathrm{EMMI})$ in the Ca II $\mathrm{K}$ line $\left(\lambda_{\mathrm{air}}=\right.$ $3933.661 \AA$ ) towards 7 QSOs located in the line-of-sight to the Magellanic Bridge. At a spectral resolution $R=\lambda / \Delta \lambda=6000$, five of the sightlines have a signal-to-noise $(S / N)$ ratio of $\sim 20$ or higher. Definite Ca absorption due to Bridge material is detected towards 3 objects, with probable detection towards two other sightlines. Gas-phase Ca II K Bridge and Milky Way abundances or lower limits for the all sightlines are estimated by the use of Parkes 21-cm H I emission line data. These data only have a spatial resolution of 14 arcmin compared with the optical observations which have milli-arcsecond resolution. With this caveat, for the three objects with sound Ca II $\mathrm{K}$ detections, we find that the ionic abundance of $\mathrm{Ca}$ II $\mathrm{K}$ relative to $\mathrm{HI}, A=\log (N(\mathrm{Ca} \mathrm{K}) / N(\mathrm{H} \mathrm{I}))$ for low-velocity Galactic gas ranges from -8.3 to -8.8 dex, with $\mathrm{H} \mathrm{I}$ column densities varying from $3-6 \times 10^{20} \mathrm{~cm}^{-2}$. For Magellanic Bridge gas, the values of $A$ are $\sim 0.5$ dex higher, ranging from $\sim 7.8$ to -8.2 dex, with $N(\mathrm{H} \mathrm{I})=1-5 \times 10^{20} \mathrm{~cm}^{-2}$. Higher values of $A$ correspond to lower values of $N(\mathrm{HI})$, although numbers are small. For the sightline towards B 0251-675, the Bridge gas has two different velocities, and in only one of these is Ca II tentatively detected, perhaps indicating gas of a different origin or present-day characteristics (such as dust content), although this conclusion is uncertain and there is the possibility that one of the components could be related to the Magellanic Stream. Higher signal-to-noise Ca II K data and higher resolution H I data are required to determine whether $A$ changes with $N(\mathrm{HI})$ over the Bridge and if the implied difference in the metalicity of the two Bridge components towards B $0251-675$ is real.

Key words. galaxies: Magellanic Clouds - galaxies: Seyfert - ISM: abundances

\section{Introduction}

The Magellanic Bridge is a filament of $\mathrm{HI}$, joining the Large and Small Magellanic Clouds (LMC, SMC), first detected and characterized by Hindman et al. (1961). This region has many interesting features, in particular that, although the gas density (generally $N(\mathrm{HI}) \sim 10^{20-21} \mathrm{~cm}^{-2}$; McGee \& Newton 1986) and metallicity (0.08 solar; Rolleston et al. 1999) are relatively low, it still contains a number of young B-type stars whose evolutionary ages and velocities make it likely that they were born in the Bridge (Hambly et al. 1994; Rolleston et al. 1999), perhaps in CO-containing clouds discovered by Muller et al. (2003a). The Bridge may have been formed by a tidal interaction between the LMC and SMC some 200 Myr ago (e.g. Sawa et al. 1999).

Previous observations of the Bridge have concentrated on its stellar content and stellar associations (e.g. Irwin et al. 1990;

^ Based on observations made with EMMI on the New Technology Telescope, La Silla, Chile, programme ID = 074.A-0038(A).
Bica \& Schmitt 1995; Graham et al. 2001), stellar abundances (Rolleston et al. 1999), H I content and dynamics (McGee \& Newton 1986; Putman et al. 1998; Kobulnicky \& Dickey 1999; Muller et al. 2003b) and CO content (Smoker et al. 2000; Muller et al. 2003a). However, to date there have only been two UV/optical interstellar absorption-line studies of the Bridge with the aim of determining the properties of the gas within it (Lehner et al. 2001; Lehner 2002). These studies used earlytype stars located in the Bridge to probe the material in the lines-of-sight. The main findings are that a wide variety of ionisation stages are present (ranging from $\mathrm{H}_{2}$ to $\mathrm{Si}$ IV and $\mathrm{C}$ IV), with the depletion patterns relative to $\mathrm{S}$ of $\mathrm{Si}, \mathrm{Fe}$ and $\mathrm{Ni}$ being very similar to that found for the Galactic halo. Although important, these studies suffer from the disadvantages that: (1) there are few sufficiently bright and blue stars within the Bridge for which optical or UV interstellar absorption measurements are possible, and (2) by their nature, it is unclear where the stars lie within the depth of the Bridge, so comparing results to $\mathrm{H}$ I emission-line observations which measure the 


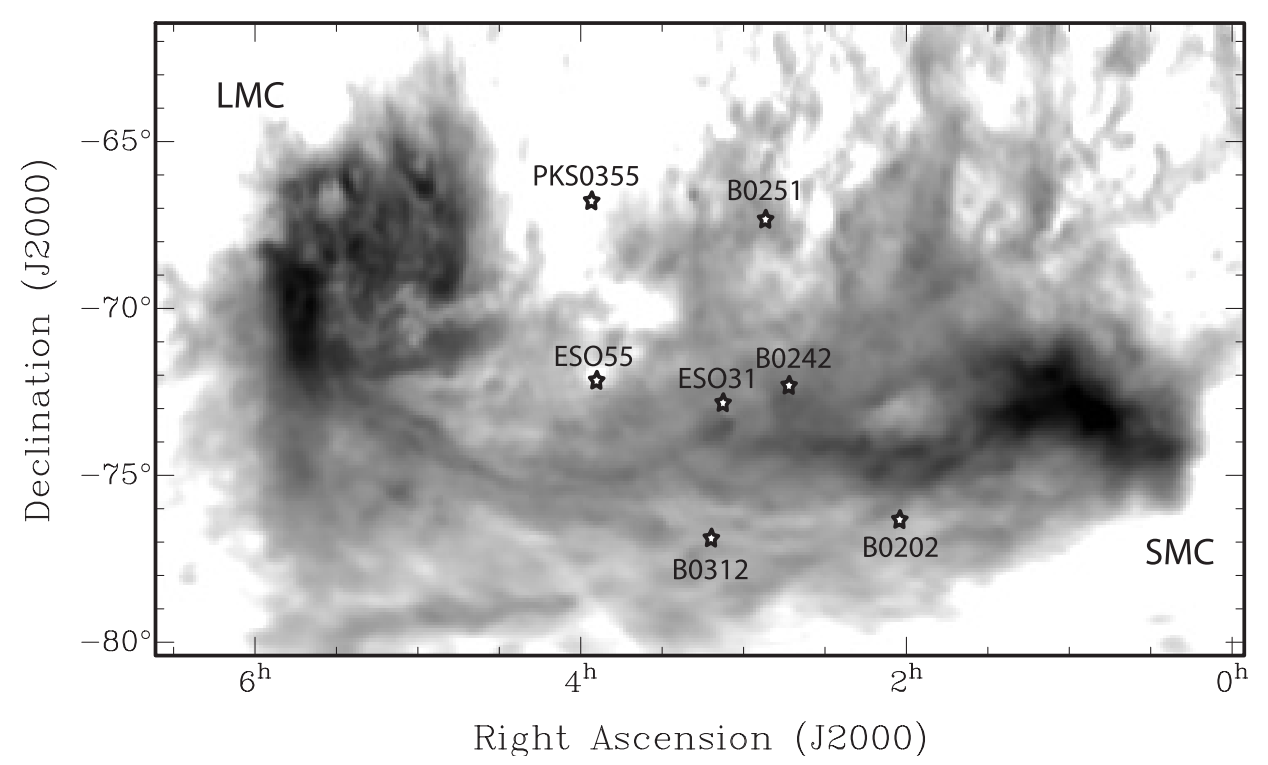

Fig. 1. Location of the QSO sample superimposed on the H I column density distribution from Brüns et al. (2005). The Ca II spectra of ESO 31-8 and PKS 0355-66 have low $S / N$ ratios.

integrated sightline is problematic. For these reasons, observing true background sources such as QSOs to determine the gas properties appears a worthwhile adventure.

The current paper aims to improve our knowledge of Bridge gas by performing Ca II K spectroscopy towards a number of background QSOs. It is a pilot study, part of whose purpose is to provide objects for future higher spectral resolution studies, as a priori one may expect that the Bridge gas will be clumpy, with some sightlines showing little or no optical or UV interstellar absorption. The clumping exists at all measured scales, and is what would be expected for a fluid which has energy dumped in at large scales, for example gravitationally (Muller et al. 2004).

The paper is organized as follows: Sect. 2 describes the sample selection. The NTT Ca II K and Parkes-ATCA H I observations and data reduction are elucidated in Sect. 3, while Sect. 4 describes the analysis of the observed Ca II K profiles and presents these spectra as well as the corresponding $\mathrm{H} \mathrm{I}$ observations. In Sect. 5 we discuss the derived parameters as a function of position within the Bridge. Finally in Sect. 6 we give the main conclusions and avenues for future research.

\section{The sample}

The sample of QSOs observed is shown in Table 1. Objects were selected from Hewitt \& Burbidge (1993) and Geha et al. (2003) and are a mix of Quasars and Seyfert galaxies. They were chosen so that they were observable with the NTT in a reasonable time, and also so that they spanned as large an angular area of the Magellanic Bridge as possible. The location of the selected objects is shown in Fig. 1 which shows the sample objects superimposed on an H I column density map of the Magellanic Clouds from Brüns et al. (2005).
Table 1. The Sample. Coordinates, redshifts and magnitudes are from SIMBAD. The Obs. time column refers to the EMMI shutter-open time in hours.

\begin{tabular}{lrrrr}
\hline \hline $\begin{array}{l}\text { QSO } \\
\text { (Type })\end{array}$ & $\begin{array}{r}\text { RA hms } \\
\text { Dec. }{ }^{\circ}{ }^{\prime \prime} \\
(\mathrm{J} 2000)\end{array}$ & $\begin{array}{r}m_{B} \\
m_{V} \\
(\mathrm{mag} .)\end{array}$ & $\begin{array}{r}\text { Obs. time } \\
(\mathrm{h})\end{array}$ \\
\hline B 0202-765 & 020213.7 & 0.39 & 16.83 & 3.75 \\
(Sy1) & -762003 & & 16.77 & \\
B 0242-7229 & 024309.6 & 0.12 & - & 2.75 \\
(Sy1) & -721648.9 & & 15.9 & \\
B 0251-675 & 025155.8 & 1.00 & - & 7.00 \\
(Quasar) & -671800 & & 17.5 & \\
ESO 31-8 & 030734.9 & 0.03 & 15.48 & 1.75 \\
(Sy1) & -725004 & & - & \\
B 0312-770 & 031155.2 & 0.22 & - & 2.25 \\
(Sy1) & -765151 & & 15.9 & \\
ESO 55-2 & 035402.0 & 0.05 & 15.67 & 3.75 \\
(Sy2) & -720804 & & - & \\
PKS 0355-66 & 035548.0 & - & 17.3 & 0.92 \\
(Quasar) & -664533.4 & & - & \\
\hline
\end{tabular}

\section{Observations and data reduction}

\subsection{NTT emmı Ca II K observations}

The optical observations described in this paper were taken during three nights in 2004 October 19-21 using the ESO Multi-Mode Instrument (EMMI) on the 3.6-m New Technology Telescope (NTT) located on La Silla, Chile. Conditions for the first two nights were clear, with observations on night three when B 0251-675 and PKS 0355-66 were observed being affected by cloud. The seeing varied from $0.5-1.6$ arcsec. The blue arm of EMMI was used with a pixel scale of 0.37 arcseconds per pixel with the holographic grating \#11 and slow readout in order to minimise readout noise (RON). The resulting RON was 5.0 electrons with a gain of 1.43 . In combination 
with a $1.5 \operatorname{arcsec}$ slit, a spectral resolution $R(=\lambda / \Delta \lambda)$ of 6000 or $50 \mathrm{~km} \mathrm{~s}^{-1}$ was obtained, centred on $3933.6 \AA$ and having a wavelength coverage of $\sim 150 \AA$. This resolution enabled us to distinguish between Galactic and Magellanic Bridge absorption, the latter being offset by $\sim+200 \mathrm{~km} \mathrm{~s}^{-1}$. ThAr arc-line spectra were observed with each target to minimise uncertainties in the wavelength scale caused by shifts in the instrument due to rotation as the objects were tracked. With the exception of PKS 0355-66, at least 3 exposures per target were taken in order to make easier the task of cosmic-ray removal. Standard reduction procedures within $\operatorname{IRAF}^{1}$ were used to produce the 1-D spectra from the 2-D images. These included bias subtraction and flatfielding using a pixel map created from dome flatfields taken during the daytime, and wavelength calibration using the attached ThAr arcs. The stability of the spectrograph was tested by checking the position of an arc line over ten images taken over three nights, and the maximum velocity shift found was $10 \mathrm{~km} \mathrm{~s}^{-1}$. However this is an upper limit in the wavelength calibration error, as the attached arcs were taken at the same rotator angle as the observations, minimising shifts caused by flexure of the instrument. Extraction of the reduced 2-D images was performed using the DosLIT package. Of the seven objects observed, five had a signal-to-noise ratio in the continuum of $\sim 20$ or above, with the remaining two objects having poorer quality spectra. After initial reduction and extraction using IRAF, the co-added Ca II $\mathrm{K}$ spectra were imported into DIPSO (Howarth et al. 1996) and converted to the kinematical Local Standard of Rest (LSR) using corrections generated by the program RV (Wallace \& Clayton 1996). The continuum was removed by fitting low-order baselines to the extracted spectra, from which we also estimated the signal to noise ratios, which range from 6-31 in the region of Ca II K. We note that the continua in the extracted spectra were relatively smooth and well-behaved with for example no obvious jumps.

\section{2. $\mathrm{H}$ I 21-cm observations}

Previously-existing 21-cm HI data were taken from two sources. One sightline towards B $0242-7229$ was previously observed by Muller et al. (2003b), and combines ATCA and Parkes data. This has a velocity channel separation of $\sim 1.6 \mathrm{~km} \mathrm{~s}^{-1}$ and a spatial resolution of 98 arcsec. Assuming a distance to the Magellanic Bridge of $\sim 55 \mathrm{kpc}$ (e.g. see Muller et al. 2003b), this corresponds to a linear scale of $\sim 25 \mathrm{pc}$ at the Bridge distance. The final spectrum has a RMS noise of 1.2 K. All seven sightlines also have Parkes-only observations of the Magellanic Bridge taken from Brüns et al. (2005). Each of these pointings is within 3 arcmin of the target QSOs. These data were taken during 1999 and used the multibeam $L$-band front-end on the Parkes radiotelescope with frequency switching. These data have a spatial resolution of $14.1 \mathrm{arcmin}$ (corresponding to $\sim 225 \mathrm{pc}$ at the distance of the Magellanic Bridge), and a velocity resolution of $0.8 \mathrm{~km} \mathrm{~s}^{-1}$. They were reduced using standard methods, which included conversion to a flux scale using observations of S8 and S9, removal of the baseline by

\footnotetext{
${ }^{1}$ IRAF is distributed by the National Optical Astronomy Observatories, USA.
}

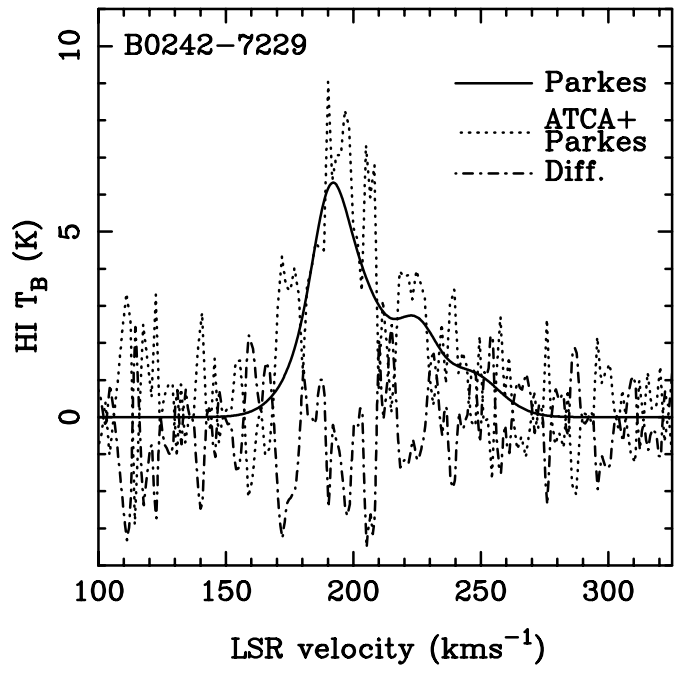

Fig. 2. Comparison between 21-cm H I ATCA-plus-Parkes observations taken from Muller et al. (2003b), and Parkes-only data taken from Brüns et al. (2005), towards the QSO B 0242-7229.

subtracting in most cases a linear baseline from the bandpasscorrected data, removal of interference spikes and conversion to the LSR.

A comparison between the ATCA-plus-Parkes and Parkesonly observations towards B 0242-7229 is shown in Fig. 2, plus the difference between the two datasets. For this particular sightline at least, and at this signal-to-noise, the difference between the profiles is negligible. The total Bridge H I column density measured using the two sightlines is similar, being $47 \times 10^{19} \mathrm{~cm}^{-2}$ for the Parkes-only data, and $52 \times 10^{19} \mathrm{~cm}^{-2}$ for the ATCA-plus-Parkes spectrum.

\section{Data analysis and results}

\subsection{Ca II K and HI spectra}

Figure 3 shows the Ca II K spectra of the seven lines of sight, plotted in the kinematical LSR. Also shown in the figure are the H I emission-line spectra described in Sect. 3.2. Immediately apparent is that the strength of Ca II $\mathrm{K}$ in the Bridge is much greater at a constant $N(\mathrm{HI})$ than in low-velocity Galactic gas. The difference was quantified by using the ELF suite of programmes within DIPSO in order to measure the equivalent widths, central velocities and velocity widths of $\mathrm{Ca}$ II $\mathrm{K}$ and $\mathrm{HI}$ components. Equivalent width results were compared for a few objects to the quick-look reduction performed during the observing run, and the results were found to be the same within 20 per cent. The results from the current reduction of the data described in Sect. 3 are those used in the results.

For Ca II K the column density was estimated from the equivalent width $(E W)$ via:

$N\left(\mathrm{~cm}^{-2}\right)=1.13 \times 10^{17} \times \frac{E W(\mathrm{~m} \AA)}{f \times \lambda^{2}(\AA)}$,

where $\lambda$ is the rest wavelength of the line and $f$ is the oscillator strength (e.g. Spitzer 1978). The Ca II K oscillator strength of 0.627 and rest wavelength of $3933.661 \AA$ were 

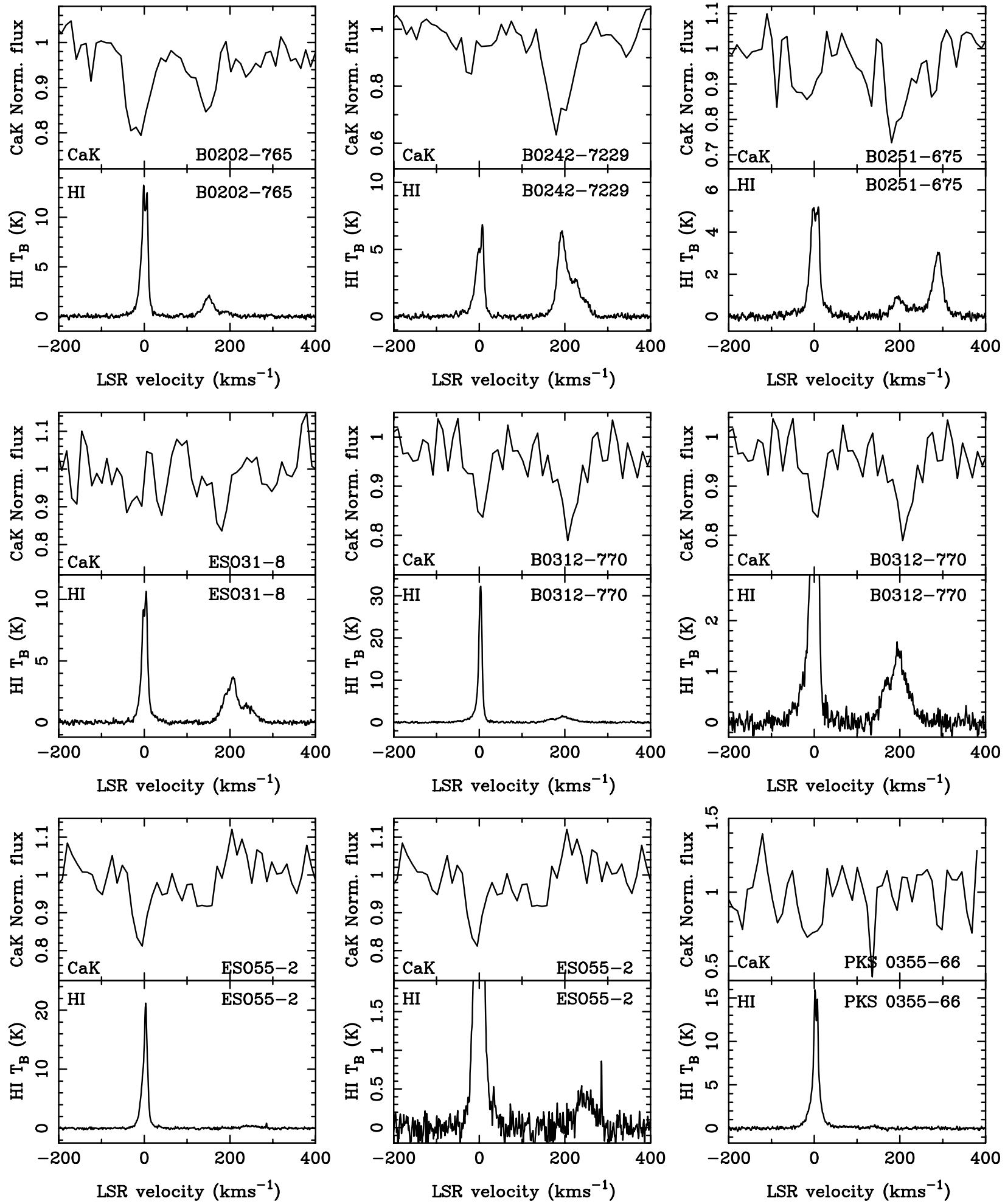

Fig. 3. Comparison between EMMI Ca II K absorption-line and Parkes 21-cm H I emission-line observations of the individual sightlines. In the cases of B 0312-770 and ESO 55-2, two plots are shown in order to highlight differences in H I strength.

taken from Morton (2003, 2004). Equation (1) is only valid in the optically-thin approximation, and hence the derived column densities are lower limits. Due to the poor velocity resolution, unresolved Ca II K absorption features will almost certainly be present.
For the H I data the column density $N(\mathrm{HI})$ was estimated thus;

$N(\mathrm{HI})\left(\mathrm{cm}^{-2}\right)=1.823 \times 10^{18} \times \int_{v 1}^{v 2} T_{\mathrm{B}} \mathrm{d} v$ 
where $T_{\mathrm{B}}$ is the brightness temperature in $\mathrm{K}$ integrated over the velocity range from $v 1$ to $v 2$ in $\mathrm{km} \mathrm{s}^{-1}$. The results from ELF and the derived column densities for the individual components are shown in Table 2, and the corresponding total column densities in Table 3.

For the sightlines with non-detections, we have estimated the limiting $\mathrm{Ca}$ II $\mathrm{K}$ equivalent width from the instrumental $F W H M$ resolution, $\Delta \lambda_{\text {inst }}=0.66 \AA$, and the $S / N$ ratio of the spectra. We can calculate the smallest $(3 \sigma)$ equivalent width, $E W_{\text {lim }}$, of an unresolved feature that we would expect to see by the relationship:

$E W_{\text {lim }}=3 \times \Delta \lambda_{\text {inst }}(S / N)^{-1}$.

These estimated limiting equivalent widths are somewhat high and are not discussed further in the paper.

Table 3 also gives the ionic abundance relative to $\mathrm{H}$, $A=$ $\log (N(\mathrm{Ca} \mathrm{K}) / N(\mathrm{H} \mathrm{I}))$. It is important to stress that the resolution of the optical and radio observations are totally different, with the Parkes 21-cm H I data sampling a much larger area than the Ca II $\mathrm{K}$ observations, so values of $A$ will clearly only be indicative, even (incorrectly) assuming that the $\mathrm{Ca} \cdot{ }^{\prime} \mathrm{II} \mathrm{K}$ spectra have no hidden substructure. With these caveats, the values of $A$ for low-velocity gas range from -8.3 to $-8.8 \mathrm{dex}$ with a range in $\mathrm{H}$ I column density from $3-6 \times 10^{20} \mathrm{~cm}^{-2}$. For Magellanic Bridge gas, the values of $A$ are $\sim 0.5$ dex higher, ranging from $\sim-7.8$ to -8.2 dex, with $N(\mathrm{H} \mathrm{I})=1-5 \times 10^{20} \mathrm{~cm}^{-2}$.

\subsection{Optical images - profile fits to observed objects}

Previously, researchers have used the extended regions in the cores of some globular clusters as a background light source to probe the structure of low and intermediate velocity interstellar clouds (IVCs) on arcsecond scales, such as the IVC towards M 15 (Meyer \& Lauroesch 1999; Smoker et al. 2002). In order to determine whether in future we could use objects in the current sample to similarly probe the gas in the Magellanic Bridge, the $B$-band acquisition images of the sample were inspected for signs of extended emission. If the objects had turned out to be extended, then follow-up observations for example using FLAMES on the VLT would be appropriate. However, only in the cases of ESO 31-8 and ESO 55-2 was there anything other than an unresolved nuclear component apparent in the images shown in Fig. 4. This was confirmed by using the Extended Surface Photometry (ESP) package (Gray et al. 2000) to fit ellipses to the main target and a nearby comparison star. The results are shown in Fig. 5 which plots the extracted counts as a function of increasing radius, using a centre fixed in $(x, y)$. Although ESO 31-8 does have a large extended component out to $\sim 5$ arcsec radius, it is a factor $\approx 100$ weaker than the nuclear peak, and would not be adequately bright for our purposes to probe the Bridge gas on arcsecond scales. ESO 55-2 may be a good candidate for follow-up projects to probe the extended absorption properties of the Bridge ISM given that its core extends to $\sim 1.8$ arcseconds with a brightness of $1 / 10$ th of the peak.

\subsection{A note on ESO55-2}

ESO 55-2 is a Seyfert 2 galaxy catalogued by Arp \& Madore (1987). It is one of only two objects in the current sample that are well-resolved by the current observations. In addition, ESO 55-2 is the only object for which we detect a clear emission line shown in Fig. 6 . This is a broad feature and lies at a (uncorrected) wavelength of $3907.91 \pm 0.06 \AA$ with a $F W H M$ width of $5.65 \pm 0.14 \AA$ (equivalent to $\sim 410 \mathrm{~km} \mathrm{~s}^{-1}$ in the restframe), and with a line flux of $4.7 \pm 0.2 \AA$. It is likely to be [O II] at $\lambda_{\text {air }}=3727.319 \AA$, redshifted to the quasar heliocentric velocity of $14538 \pm 74 \mathrm{~km} \mathrm{~s}^{-1}$ (Sekiguchi \& Wolsentcroft 1993).

\section{Discussion}

There are limitations on the information concerning the physical conditions of the gas in the Bridge from the Ca II K and H I spectra alone, especially where they have such different spatial resolutions. The main result is that in the three sightlines with reasonably well-defined values of the Magellanic Bridge Ca II $\mathrm{K}$ absorption, the values of $A$ are some $\sim 0.5$ dex higher than in Galactic gas. We recall that Wakker \& Mathis (2000) (although see Smoker et al. 2003) related Ca II K to H I in the following way;

$\log A(\mathrm{Ca}$ II $)=-0.78 \times(\log (N(\mathrm{H} \mathrm{I}))-19.5)-7.78$.

Hence for typical Galactic gas which has $N(\mathrm{HI})$ of $\sim 5 \times$ $10^{20} \mathrm{~cm}^{-2}$, we would expect $A(\mathrm{Ca}$ II $) \sim-8.7$, which is as observed in the current sightlines. If the physical properties of Bridge gas were the same as for Galactic gas, for the Bridge sightlines which have $N(\mathrm{H} \mathrm{I})$ of $\sim 2 \times 10^{20} \mathrm{~cm}^{-2}$, we would expect $A(\mathrm{Ca}$ II $) \sim-8.4$, which is a smaller by $\sim 0.5$ dex than is observed in the current Bridge dataset. The difference could be caused by a number of conflicting factors including; (a) ionisation issues. If some of the hydrogen is in ionised form in the Bridge this would cause $N(\mathrm{HI})$ to be lower in the Bridge and hence $A$ to be higher. Observations in $\mathrm{H} \alpha$ would be able to determine the level of ionisation of the hydrogen in the Bridge and Galaxy components on the current sightlines; (b) the presence of less dust in the Magellanic Bridge than in the Milky Way onto which $\mathrm{Ca}$ is bound, which causes less depletion and hence would increase the observed value of $N(\mathrm{Ca}$ II $)$ and $A$; and (c) a smaller intrinsic metallicity in the Magellanic Bridge system when compared with the Milky Way which would decrease both $N(\mathrm{Ca}$ II) and $A$. Previous work on a Magellanic Bridge B-type star by Rolleston et al. (1999) and interstellar observations of the Bridge by Lehner et al. (2001) support a metallicity of $\sim-1.1$ dex compared to the Galaxy. Figure 7 displays $A(\mathrm{Ca}$ II K) against $N(\mathrm{HI})$ for the low-velocity and Bridge gas respectively. Also plotted on the diagram is the best-fitting line taken from Wakker \& Mathis (2000). The current low velocity points scatter about this best-fitting line. As noted above, the Magellanic Bridge points are offset by about 0.5 dex, although to first order the slope of the line (admittedly heavily biased by the observation towards B 0242-7229) seems to be the same as for the low-velocity gas. 
Table 2. Comparison between H I emission-line and Ca II K absorption line data.

\begin{tabular}{|c|c|c|c|c|c|c|c|}
\hline $\begin{array}{l}\text { QSO } \\
\text { sightline }\end{array}$ & $\begin{array}{r}v_{\mathrm{HI}} \\
\left(\mathrm{km} \mathrm{s}^{-1}\right)\end{array}$ & $\begin{array}{r}F W H M(\mathrm{HI}) \\
\left(\mathrm{km} \mathrm{s}^{-1}\right)\end{array}$ & $\begin{array}{r}T_{B}^{\max }(\mathrm{HI}) \\
(\mathrm{K})\end{array}$ & $\begin{array}{r}\text { H I flux } \\
\left(\mathrm{K} \mathrm{km} \mathrm{s}^{-1}\right)\end{array}$ & $\begin{array}{r}v_{\mathrm{CaK}} \\
\left(\mathrm{km} \mathrm{s}^{-1}\right)\end{array}$ & $\begin{array}{r}E W(\mathrm{CaK}) \\
(\mathrm{m} \AA)\end{array}$ & $\begin{array}{r}S / N \text { ratio } \\
(\mathrm{CaK})\end{array}$ \\
\hline B 0202-765 & $-4.9 \pm 0.6$ & $30.1 \pm 1.2$ & $2.27 \pm 0.26$ & $72.9 \pm 6.1$ & $-14.7 \pm 3.1$ & $174.5 \pm 18.0$ & 31 \\
\hline “ & $0.4 \pm 0.1$ & $14.8 \pm 0.3$ & $8.68 \pm 0.25$ & $137.3 \pm 6.5$ & - & - & \\
\hline “ & $-1.6 \pm 0.0$ & $2.7 \pm 0.1$ & $2.91 \pm 0.11$ & $8.4 \pm 0.5$ & - & - & \\
\hline “ & $6.7 \pm 0.0$ & $4.1 \pm 0.1$ & $5.34 \pm 0.12$ & $23.6 \pm 1.0$ & - & - & \\
\hline “ & $150.0 \pm 0.2$ & $28.0 \pm 0.6$ & $1.86 \pm 0.03$ & $55.8 \pm 1.0$ & $+139.2 \pm 3.5$ & $122.1 \pm 15.4$ & \\
\hline “" & $190.2 \pm 0.8$ & $22.4 \pm 2.3$ & $0.45 \pm 0.03$ & $10.8 \pm 0.9$ & - & - & \\
\hline В $0242-7229$ & $-35.6 \pm 0.0$ & $22.7 \pm 0.0$ & $0.39 \pm 0.02$ & $9.4 \pm 0.6$ & $-24.1 \pm 2.9$ & $50.1 \pm 16.4$ & 21 \\
\hline “ & $-0.3 \pm 0.0$ & $22.7 \pm 0.0$ & $4.75 \pm 0.02$ & $115.2 \pm 0.7$ & - & - & \\
\hline “ & $7.6 \pm 0.0$ & $5.6 \pm 0.0$ & $3.40 \pm 0.05$ & $20.6 \pm 0.3$ & - & - & \\
\hline “ & $190.5 \pm 0.2$ & $14.6 \pm 0.7$ & $2.40 \pm 0.20$ & $37.5 \pm 4.7$ & $+185.7 \pm 2.5$ & $282.8 \pm 24.9$ & \\
\hline “ & $196.6 \pm 0.4$ & $33.6 \pm 0.9$ & $4.19 \pm 0.19$ & $150.3 \pm 3.7$ & - & - & \\
\hline “ & $225.0 \pm 0.0$ & $18.0 \pm 0.6$ & $1.81 \pm 0.08$ & $34.8 \pm 2.1$ & - & - & \\
\hline “ & $245.0 \pm 0.0$ & $27.9 \pm 0.9$ & $1.20 \pm 0.03$ & $35.7 \pm 1.1$ & - & - & \\
\hline В $0251-675$ & $2.1 \pm 0.7$ & $56.0 \pm 3.8$ & $0.86 \pm 0.10$ & $51.8 \pm 3.4$ & $-21.6 \pm 7.4$ & $120.1 \pm 32.3$ & 19 \\
\hline “ & $9.7 \pm 0.1$ & $7.0 \pm 0.4$ & $2.83 \pm 0.20$ & $21.2 \pm 2.8$ & - & - & \\
\hline “ & $-1.3 \pm 0.3$ & $17.7 \pm 0.9$ & $4.24 \pm 0.10$ & $80.2 \pm 6.0$ & - & - & \\
\hline “ & $194.4 \pm 0.5$ & $26.7 \pm 1.8$ & $0.68 \pm 0.03$ & $19.6 \pm 1.8$ & $+194.5 \pm 9.1$ & $268.8 \pm 52.5$ & \\
\hline “ & $250.9 \pm 4.5$ & $110.9 \pm 6.3$ & $0.39 \pm 0.01$ & $46.2 \pm 2.7$ & - & - & \\
\hline “ & $288.7 \pm 0.1$ & $24.4 \pm 0.4$ & $2.64 \pm 0.03$ & $68.8 \pm 1.4$ & - & - & \\
\hline В $0312-770$ & $2.8 \pm 0.0$ & $7.8 \pm 0.0$ & $29.84 \pm 0.25$ & $249.8 \pm 3.5$ & $2.1 \pm 4.1$ & $87.1 \pm 18.8$ & 30 \\
\hline “ & $-4.8 \pm 0.4$ & $9.0 \pm 0.8$ & $2.94 \pm 0.19$ & $28.5 \pm 4.3$ & - & - & \\
\hline “" & $-4.6 \pm 0.3$ & $37.8 \pm 1.3$ & $2.13 \pm 0.12$ & $86.2 \pm 2.4$ & - & - & \\
\hline “ & $+168.3 \pm 1.0$ & $28.4 \pm 8.0$ & $0.15 \pm 0.07$ & $4.6 \pm 1.5$ & $+208.5 \pm 4.2$ & $141.8 \pm 23.2$ & \\
\hline “ & $+168.3 \pm 2.0$ & $10.0 \pm 1.0$ & $0.13 \pm 0.08$ & $1.4 \pm 0.5$ & - & - & \\
\hline “ & $+195.8 \pm 1.0$ & $64.0 \pm 2.1$ & $0.87 \pm 0.05$ & $59.3 \pm 2.7$ & - & - & \\
\hline “" & $+197.2 \pm 0.6$ & $17.4 \pm 2.0$ & $0.55 \pm 0.06$ & $10.1 \pm 1.9$ & - & - & \\
\hline ESO $31-8$ & $-2.5 \pm 0.0$ & $5.9 \pm 0.2$ & $4.47 \pm 0.15$ & $28.5 \pm 1.8$ & - & $<200$ & 10 \\
\hline “ & $-0.7 \pm 0.1$ & $22.5 \pm 0.4$ & $4.45 \pm 0.16$ & $106.8 \pm 2.5$ & - & " & \\
\hline “ & $4.4 \pm 0.0$ & $6.1 \pm 0.1$ & $6.59 \pm 0.12$ & $42.9 \pm 1.5$ & - & $"$ & \\
\hline “" & $28.6 \pm 1.3$ & $21.2 \pm 3.1$ & $0.32 \pm 0.03$ & $7.3 \pm 1.0$ & - & $"$ & \\
\hline “ & $198.0 \pm 1.0$ & $35.7 \pm 1.1$ & $2.52 \pm 0.08$ & $96.2 \pm 5.9$ & $+179.5 \pm 5.0$ & $75.0 \pm 40.0$ & \\
\hline “ & $208.8 \pm 0.1$ & $11.8 \pm 0.8$ & $1.46 \pm 0.11$ & $18.4 \pm 2.4$ & - & - & \\
\hline “" & $241.1 \pm 1.4$ & $40.3 \pm 2.2$ & $1.21 \pm 0.03$ & $51.9 \pm 3.8$ & - & - & \\
\hline ESO 55-2 & $-0.8 \pm 0.4$ & $14.0 \pm 0.7$ & $7.04 \pm 0.64$ & $105.2 \pm 9.9$ & $-8.8 \pm 3.6$ & $91.2 \pm 20.1$ & 19 \\
\hline “ & $-1.2 \pm 0.3$ & $29.6 \pm 1.7$ & $2.49 \pm 0.43$ & $78.8 \pm 9.3$ & - & - & \\
\hline “ & $2.9 \pm 0.0$ & $3.4 \pm 0.1$ & $4.39 \pm 0.27$ & $16.2 \pm 1.6$ & - & - & \\
\hline “ & $4.3 \pm 0.1$ & $8.2 \pm 0.2$ & $9.44 \pm 0.68$ & $82.4 \pm 8.0$ & - & - & \\
\hline “ & $35.3 \pm 0.6$ & $8.8 \pm 2.0$ & $0.36 \pm 0.05$ & $3.4 \pm 0.5$ & - & - & \\
\hline “ & $246.7 \pm 1.1$ & $45.4 \pm 2.8$ & $0.39 \pm 0.02$ & $19.1 \pm 1.0$ & - & $<100$ & \\
\hline PKS 0355-66 & $-16.0 \pm 2.1$ & $21.5 \pm 3.2$ & $0.80 \pm 0.10$ & $18.5 \pm 4.7$ & - & $<330$ & 6 \\
\hline “ & $3.9 \pm 0.2$ & $21.2 \pm 0.4$ & $6.78 \pm 0.17$ & $153.2 \pm 4.9$ & - & " & \\
\hline “ & $1.6 \pm 0.0$ & $5.0 \pm 0.0$ & $9.00 \pm 0.13$ & $48.6 \pm 1.2$ & - & $"$ & \\
\hline “ & $7.3 \pm 0.0$ & $3.5 \pm 0.0$ & $7.78 \pm 0.12$ & $29.8 \pm 0.8$ & - & $"$ & \\
\hline “ & $25.9 \pm 4.2$ & $125.9 \pm 8.6$ & $0.31 \pm 0.02$ & $42.0 \pm 2.4$ & - & $"$ & \\
\hline
\end{tabular}

Concluding, the observed value of $A$ is affected by many variables, and the current data alone are not sufficient to unscramble the different effects.

One sightline, B 0251-675, is particularly interesting and merits further study. This position lies approximately mid-way between the SMC and LMC and has two clear velocity components visible in $\mathrm{HI}$ at $\sim+195$ and $+289 \mathrm{~km} \mathrm{~s}^{-1}$, joined by a Bridge of gas with velocity $\approx+249 \mathrm{~km} \mathrm{~s}^{-1}$. Although the line flux of the $+289 \mathrm{~km} \mathrm{~s}^{-1}$ component is some 3.7 times stronger than that at $195 \mathrm{~km} \mathrm{~s}^{-1}$, Fig. 3 shows obvious absorption only in the lower velocity component. The H I component at $+289 \mathrm{~km} \mathrm{~s}^{-1}$ has a line flux of $69 \mathrm{~K} \mathrm{~km} \mathrm{~s}^{-1}$ or $1.3 \times 10^{20} \mathrm{~cm}^{-2}$. 
Table 3. Comparison of H I emission-line and Ca II K absorption line data - total column densities. "Bridge" refers to Magellanic Bridge gas and "LV" to low-velocity gas. As noted in the text, there are almost certainly unresolved Ca II components, these values are lower limits. The Ca II K detections towards B 0251-675 and ESO 31-8 are tentative.

\begin{tabular}{|c|c|c|c|c|c|c|}
\hline$\overline{Q S O}$ & Region & $\begin{array}{r}\mathrm{HI} \text { I(Tot) } \\
\mathrm{K} \mathrm{km} \mathrm{s}^{-1} \\
\end{array}$ & $\begin{array}{r}\mathrm{CaK}(\mathrm{Tot}) \\
\mathrm{m} \AA\end{array}$ & $\begin{array}{r}N(\mathrm{H} \mathrm{I}) \\
\times 10^{19} \mathrm{~cm}^{-2} \\
\end{array}$ & $\begin{array}{r}N(\mathrm{CaK}) \\
\times 10^{11 \mathrm{~cm}^{-2}} \\
\end{array}$ & $A(\mathrm{CaK})$ \\
\hline $\begin{array}{l}\text { В } 0202-765 \\
\text { “ }\end{array}$ & $\begin{array}{r}\text { LV } \\
\text { Bridge }\end{array}$ & $\begin{array}{r}242.2 \pm 10.2 \\
66.6 \pm 1.9\end{array}$ & $\begin{array}{l}174.5 \pm 18.0 \\
122.1 \pm 15.4\end{array}$ & $\begin{array}{l}44.2 \pm 1.9 \\
12.1 \pm 0.4\end{array}$ & $\begin{array}{l}20.3 \pm 2.1 \\
14.2 \pm 1.8\end{array}$ & $\begin{array}{l}-8.33 \pm 0.06 \\
-7.93 \pm 0.07\end{array}$ \\
\hline $\begin{array}{l}\text { В 0242-7229 } \\
\text { “ }\end{array}$ & $\begin{array}{r}\text { LV } \\
\text { Bridge }\end{array}$ & $\begin{array}{l}145.2 \pm 3.1 \\
258.3 \pm 8.2\end{array}$ & $\begin{array}{r}50.1 \pm 16.4 \\
282.8 \pm 24.9\end{array}$ & $\begin{array}{l}26.5 \pm 0.6 \\
47.1 \pm 1.5\end{array}$ & $\begin{array}{r}5.8 \pm 1.9 \\
33.0 \pm 2.9\end{array}$ & $\begin{array}{l}-8.65 \pm 0.12 \\
-8.15 \pm 0.05\end{array}$ \\
\hline $\begin{array}{l}\text { В } 0251-675 \\
\text { “ }\end{array}$ & $\begin{array}{r}\text { LV } \\
\text { Bridge }\end{array}$ & $\begin{array}{l}153.2 \pm 8.0 \\
134.6 \pm 4.5\end{array}$ & $\begin{array}{l}120.1 \pm 32.3 \\
268.8 \pm 52.5\end{array}$ & $\begin{array}{l}27.9 \pm 1.5 \\
24.5 \pm 0.8\end{array}$ & $\begin{array}{l}14.0 \pm 3.8 \\
31.3 \pm 6.1\end{array}$ & $\begin{array}{l}-8.30 \pm 0.13 \\
-7.89 \pm 0.09\end{array}$ \\
\hline $\begin{array}{l}\text { В } 0312-770 \\
\text { “ }\end{array}$ & $\begin{array}{r}\text { LV } \\
\text { Bridge }\end{array}$ & $\begin{array}{r}364.5 \pm 9.4 \\
75.4 \pm 4.0\end{array}$ & $\begin{array}{r}87.1 \pm 18.8 \\
208.5 \pm 23.2\end{array}$ & $\begin{array}{l}66.4 \pm 1.7 \\
13.7 \pm 0.7\end{array}$ & $\begin{array}{l}10.1 \pm 2.2 \\
24.3 \pm 2.7\end{array}$ & $\begin{array}{l}-8.81 \pm 0.09 \\
-7.75 \pm 0.07\end{array}$ \\
\hline $\begin{array}{l}\text { ESO 31-8 } \\
\text { “ }\end{array}$ & $\begin{array}{r}\text { LV } \\
\text { Bridge }\end{array}$ & $\begin{array}{r}185.5 \pm 16.8 \\
166.5 \pm 8.3\end{array}$ & $75.0 \pm 40.0$ & $\begin{array}{l}33.8 \pm 3.1 \\
30.4 \pm 1.6\end{array}$ & $\begin{array}{r}<23.0 \\
8.7 \pm 4.6\end{array}$ & $\begin{array}{r}<-8.16 \\
-8.58 \pm 0.25\end{array}$ \\
\hline $\begin{array}{l}\text { ESO 55-2 } \\
\text { “ }\end{array}$ & $\begin{array}{r}\text { LV } \\
\text { Bridge }\end{array}$ & $\begin{array}{r}286.0 \pm 16.2 \\
19.1 \pm 1.1\end{array}$ & $\begin{array}{r}91.2 \pm 20.1 \\
-\end{array}$ & $\begin{array}{r}52.1 \pm 3.0 \\
3.4 \pm 0.2\end{array}$ & $\begin{array}{r}10.6 \pm 2.4 \\
<12.0\end{array}$ & $\begin{array}{r}-8.68 \pm 0.10 \\
<-7.45\end{array}$ \\
\hline $\begin{array}{l}\text { PKS 0355-66 } \\
\text { “ }\end{array}$ & $\begin{array}{r}\text { LV } \\
\text { Bridge }\end{array}$ & $\begin{array}{r}292.1 \pm 9.4 \\
-\end{array}$ & - & $\begin{array}{r}53.2 \pm 1.8 \\
-\end{array}$ & $\begin{array}{r}<38.4 \\
"\end{array}$ & $\begin{array}{r}<-8.13 \\
-\end{array}$ \\
\hline
\end{tabular}
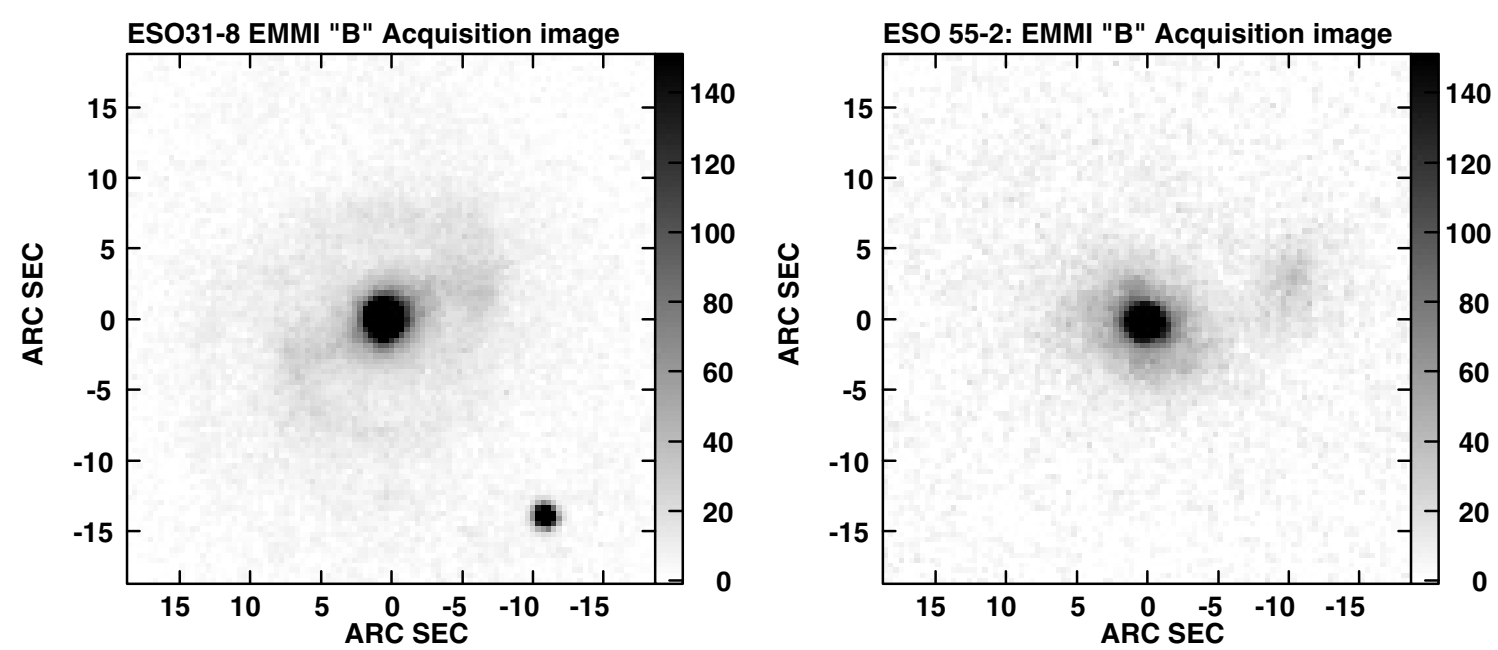

Fig. 4. EMMI $B$-band acquisition images of two extended objects in the sample. North is up and East is to the left. The peak level for the ESO 31-8 image in the Seyfert nucleus is 5200 counts above sky and for ESO 55-2 is 820 counts above sky.

This could imply that at this position, the Bridge is composed of gas which has different origins, or currently has different ionisation or dust properties.

It appears that towards the B 0251-675 sightline, we could be probing two different "arms" of the SMC, Bridge or Magellanic Stream. The two components are separated by a few kpc and are both visible in HI. The lower velocity arm is obviously turbulent and morphologically complex (Muller et al. 2004), and numerical simulations by Gardiner et al. (1994) suggest this component forms the actual "Bridge" link between the SMC and LMC. The higher velocity component is an almost radially extending arm, and has a much smoother appearance in $\mathrm{HI}$.

The continued tidal interactions between the Magellanic Clouds is the most likely mechanism for the mixing of the $\mathrm{HI}$ in the Magellanic Bridge. This feature is also apparently more chemically enriched, relative to the higher velocity component, as suggested in the numerical simulations by Gardiner et al. (1994) and by our EMMI observations.

Higher $S / N$ ratio Ca II data combined with high angularresolution $\mathrm{HI}$ observations would be needed to confirm the current finding because there remains the possibility that there is H I gas towards the B 0251-675 that may only be detected 

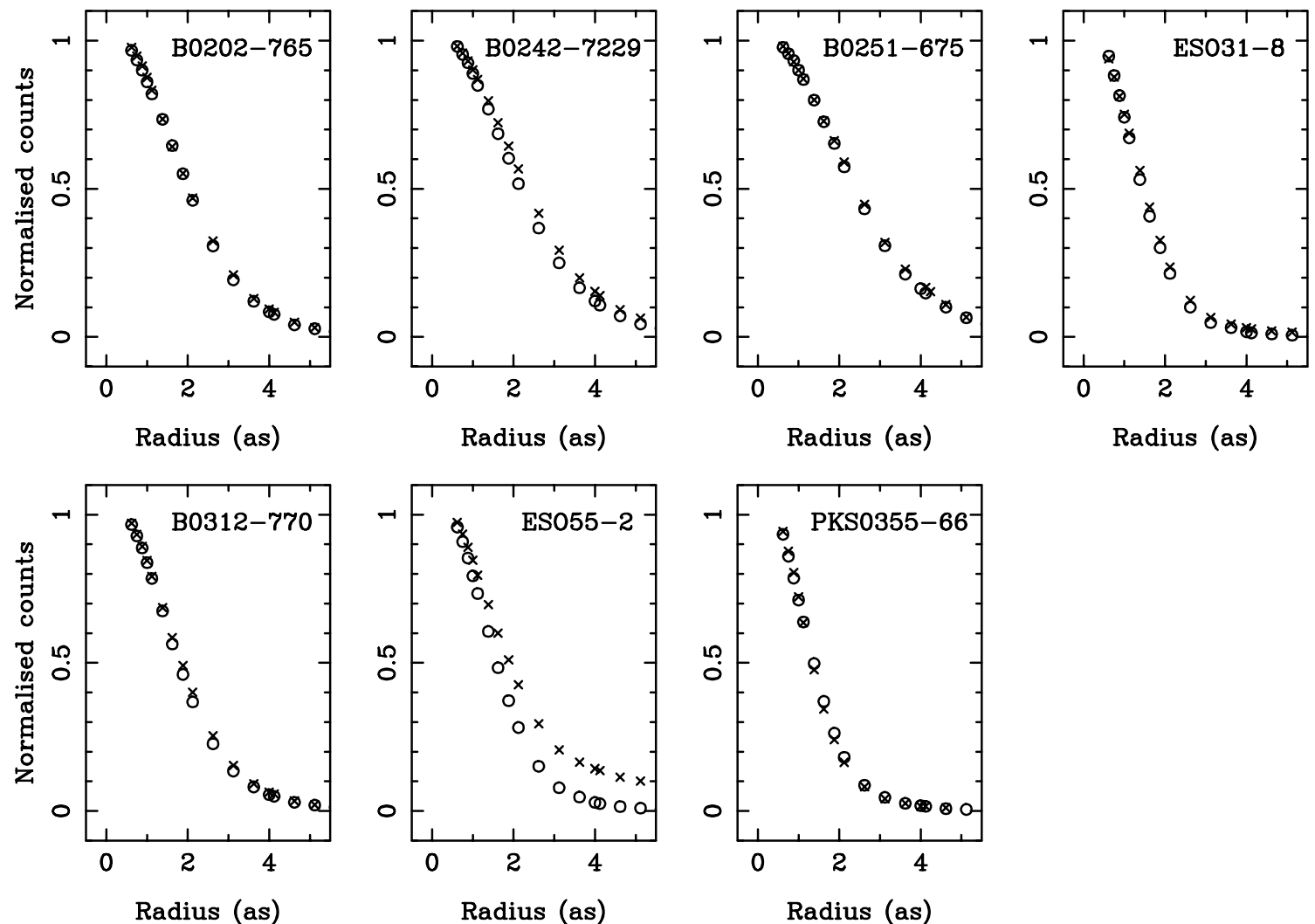

Fig. 5. EMMI $B$-band acquisition images: profile fits to the sample objects. Open circles refer to fits to nearby stars and crosses to fits to the QSOs. When the points lie on top of each other this implies that the QSOs are unresolved by the current observations.

using a pencil beam where at present it is diluted by the large Parkes resolution. Higher resolution observations are thus necessary to more convincingly associate the absorption feature with a line of sight $\mathrm{H}$ I component.

\section{Conclusions and future work}

We have presented intermediate spectral resolution Ca II K and high spectral resolution H I observations towards 7 QSO sightlines in the Magellanic Bridge. Clearly the current observations have provided only a first-look towards QSO Bridge sightlines, as the signal-to-noise ratios in the Ca II $\mathrm{K}$ spectra are at the limit for what can be usefully derived and the optical and $\mathrm{HI}$ data are not well matched in resolution. In the three objects with good $S / N$ ratio, we find that the ionic abundance of $\mathrm{Ca}$ II $\mathrm{K}$ relative to $\mathrm{H}$ I is $\sim 0.5$ dex higher than for local gas, assuming that the H I column density on arcsecond scales matches that at the Parkes resolution of 14 arcmin and of course in the absence of ionisation corrections or differences in dust content.

Obvious ground-based follow-up observations to the present work would be higher-resolution spectroscopy in $\mathrm{Ca}$ II $\mathrm{K}$ and the $\mathrm{Na} \mathrm{D}$ lines on a 8-m telescope, for example using UVES on the VLT. This would both enable a higher $S / N$ to be attained, and also to easily distinguish different Bridge components, especially towards B 0251-675, determining whether the apparent difference in the abundance observed in the two velocity components is in fact real. Finally, with very good seeing,

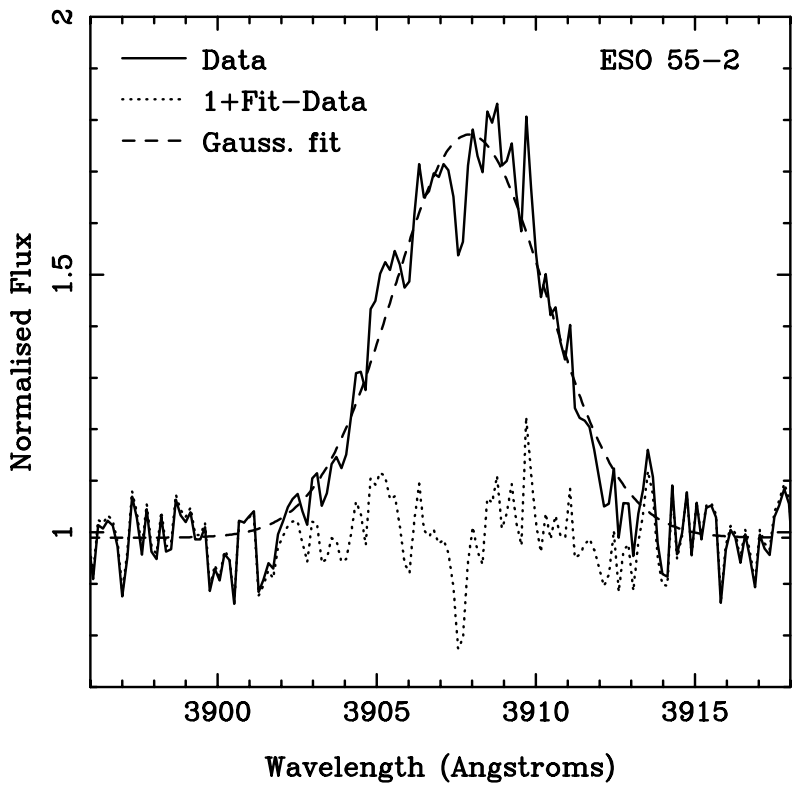

Fig. 6. ESO 55-2; normalised EMMI spectrum in the region of redshifted [OII] $\left(\lambda_{\text {air }}=3727.319 \AA\right)$. One plus the residual of the Gaussian-fit minus the extracted spectrum is shown as a dotted line.

the ARGUS mode of FLAMES could be used to probe the arcsecond scale structure of Bridge gas, using the Sy2 ESO 55-2 as a background source. 


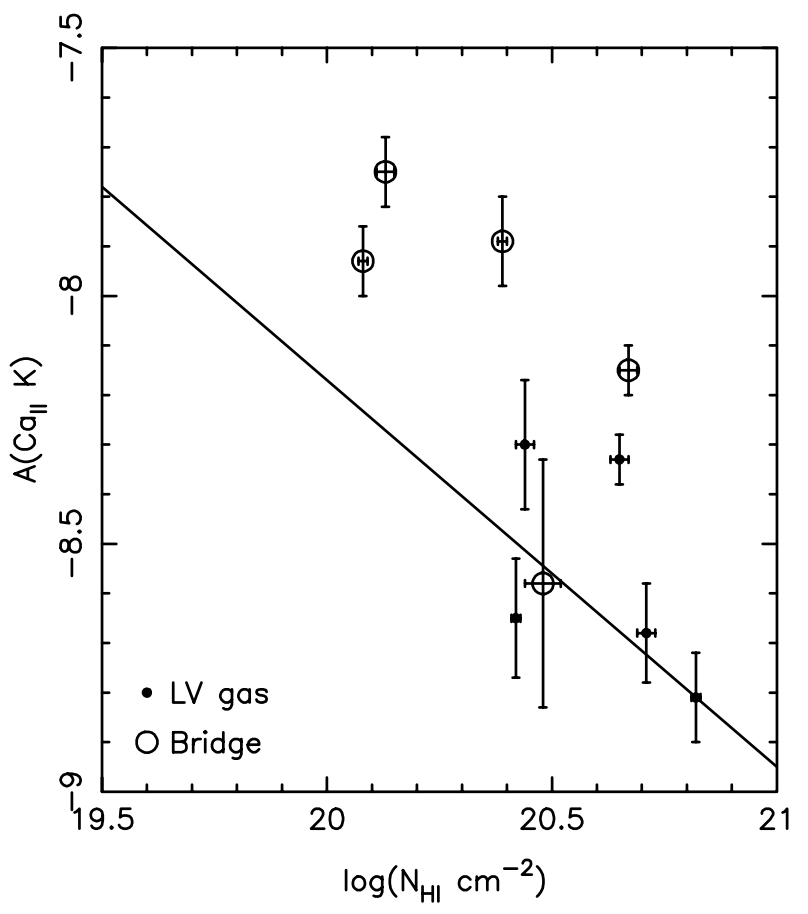

Fig. 7. Comparison between $A(\mathrm{Ca}$ II $)$ and $\log \left(N_{\mathrm{HI}}\right)$ for the low-velocity and Bridge components in the current dataset. The solid line refers to the best fitting value taken from Wakker \& Mathis (2000). We note that the Ca II abundances are likely lower limits due to the fact that unresolved features will almost certainly be present in the EMMI data.

Acknowledgements. We acknowledge the help of Chip Kobulnicky in preparing the original proposal. H.M.A.T. and I.H. acknowledge financial support from the Department for Education and Learning for Northern Ireland, while J.K.L. is supported by the Particle Physics and Astronomy Research Council of the United Kingdom. F.P.K. is grateful to AWE Aldermaston for the award of a William Penney Fellowship. This research has made use of the SIMBAD database, operated at CDS, Strasbourg, France. We would like to thank Emanuela Pompei and Cedric Foellmi for support during our enjoyable run at La Silla. We thank an anonymous referee for their useful suggestions for improvement to the paper.

\section{References}

Arp, H. C., \& Madore, B. F. 1987, A catalogue of Southern Peculiar Galaxies and Associations (Cambridge University Press)

Bica, E. L. D., \& Schmitt, H. R. 1995, ApJS, 101, 41
Brüns, C., Kerp, J., Staveley-Smith, L., et al. 2005, A\&A, 432, 45

Gardiner, L. T., Sawa, T., \& Fujimoto, M. 1994, MNRAS, 266, 567

Geha, M., Alcock, C., Allsman, R. A., et al. 2003, AJ, 125, 1

Graham, M. F., Smith, R. J., Meaburn, J., \& Bryce, M. 2001, MNRAS, 326,539

Gray, N., Taylor, M., \& Privett, G. 2000, STARLINK, User Note SUN 180.5, Rutherford Appleton Laboratory/CCLRC

Hambly, N. C., Dufton, P. L., Keenan F. P., et al. 1994 A\&A, 285, 716

Hewitt, A., \& Burbidge, G. 1993, ApJS, 87, 451

Hindman, J. V., McGee, R. X., Carter, A. W. L., \& Kerr F. J. 1961, AJ, 66,45

Howarth, I. D., Murray, J., Mills, D., \& Berry, D. S. 1996, STARLINK, User Note SUN 50, Rutherford Appleton Laboratory/CCLRC

Irwin, M. J., Demers, S., \& Kunkel W. E. 1990, AJ, 99, 191

Kobulnicky, H. A., \& Dickey, J. M. 1999, AJ, 117, 908

Lehner, N., Sembach, K. R., Dufton, P. L., Rolleston, W. R. J., \& Keenan, F. P. 2001, ApJ, 551, 781

Lehner, N., 2002, ApJ, 578, 126

McGee, R. X., \& Newton, L. M. 1986, Proc. Astron. Soc. Aust, 6, 471

Meyer, D. M., \& Lauroesch, J. T. 1999, ApJ, 520, 103

Morton, D. C. 2003, ApJS, 149, 205

Morton, D. C. 2004, ApJS, 151, 403

Muller, E., Staveley-Smith, L., \& Zealey, W. J. 2003a, MNRAS, 338, 609

Muller, E., Staveley-Smith, L., Zealey, W., \& Stanimirovic, S. 2003b, MNRAS, 339, 105

Muller, E., Stanimirovic, S., Rosolowsky, E., \& Staveley-Smith, L. 2004, ApJ, 616, 845

Putman, M. E., Gibson, B. K., Staveley-Smith, L., et al. 1998, Nature, 394, 752

Rolleston, W. R. J., Dufton, P. L., McErlean, N. D., \& Venn, K. A. 1999, A\&A, 348, 728

Sawa, T., Fujimoto, M., \& Kumai Y. 1999, in New Views of the Magellanic Clouds. Astron. Soc. Pac. San Francisco, ed. Y.-H. Chu, N. B. Suntzeff, J. E. Hesser, D. A. Bohlender, Proc. IAU Symp., 190, 499

Sekiguchi, K., \& Wolstencroft, R. D. 1993, MNRAS, 263, 349

Smoker, J. V., Keenan, F. P., Polatidis, A. G., Mooney, C. J., \& Lehner, N. 2000, A\&A, 463, 451

Smoker, J. V., Haffner, L. M., Keenan, F. P., Davies, R. D., \& Pollacco, D. 2002, MNRAS, 337, 385

Smoker, J. V., Rolleston, W. R. J., Kay, H. R. M., et al. 2003, MNRAS, 346, 119

Spitzer, L. 1978, Physical processes in the interstellar medium (New York: Wiley)

Wakker, B. P., \& Mathis, J. S. 2000, ApJ, 544, 107

Wallace, P., \& Clayton, C. 1996, RV, STARLINK, User Note SUN 78, Rutherford Appleton Laboratory/CCLRC 